\title{
Abordaje y afrontamiento del estrés en el post-COVID: Equipos de Protección Emocional (EPE) en el "Consorci Sanitari Integral (CSI)"
}

\author{
Elisenda Reig Puig ${ }^{1}$, Rosario Arias ${ }^{2}$, Montse Vacas 3 , \\ Xavi Canadell ${ }^{4}$ y Mónica Prat 5 \\ L'Hospitalet de Llobregat (Barcelona)
}

La pandemia puso a los profesionales sanitarios en riesgo de trastorno por estrés postraumático. La dificultad de ayudarles en plena crisis, obligó a movilizar recursos y ofrecer estrategias psicológicas para afrontar el trauma de la pandemia en un espacio de tiempo muy corto.

La unidad de psiquiatría coordinó un equipo de profesionales (psiquiatría / psicología) para apoyar, mediante terapia grupal, al personal sanitario que lo requirió, así como para dar soporte al duelo a los que habían perdido a un familiar en nuestros centros de asistencia. Nuestra intervención, durante y en el post-covid, demuestra que la terapia grupal, en distintos formatos, es una posibilidad interesante para asistir a muchas personas al mismo tiempo y con buenos resultados. La relación coste-beneficio es muy eficiente, dado el gran número de personas asistidas (449 personas) respecto al número de profesionales implicados (8 psicólogos). Presentamos en este artículo el Proyecto Equipos de protección emocional (EPE) desarrollado en nuestro centro después de la fase de crisis de la pandemia.

Palabras clave: Terapia Grupal, estrés postraumático, soporte psicológico, pandemia covid.

The pandemic put healthcare professionals at risk for post-traumatic stress disorder. The difficulty of helping them in the midst of the crisis forced them to mobilize resources and offer psychological strategies to face the trauma of the pandemic.

The Psychiatry unit coordinated a team of professionals (psychiatry / psychology) to support, through group therapy, the health personnel who required it, as well as to support the grief of those who had lost a family member in our assistance centers. Our intervention, during and in the post-covid period, shows that group therapy, in different formats, is an interesting possibility to assist many people at the same time and with good results. The cost-benefit ratio is very efficient, given the large number of people assisted (449 people) compared to the number of professionals involved (8 psychologists). We present in this article the Emotional Protection Equipment (EPE) Project developed in our center after the crisis phase of the pandemic.

Key Words: Group Therapy, Post-traumatic stress, Psychological support, Covid pandemic.

English Title: Addressing and coping with stress in post-COVID: Emotional Protection Equipment (EPE) in the "Consorci Sanitari Integral (CSI)"

\section{Cita bibliográfica / Reference citation:}

Reig, E., Arias, R., Vacas, M., Canadell, X. y Prat, M. (2021). Abordaje y afrontamiento del estrés en el post-COVID: Equipos de Protección Emocional (EPE) en el "Consorci Sanitari Integral (CSI)". Clínica e Investigación Relacional, 15 (2): 402-418. [ISSN 1988-2939] [Recuperado de www.ceir.info ] DOI: 10.21110/19882939.2021.150207

\footnotetext{
${ }^{1}$ Reig Puig, E. Psicóloga Clínica adjunta Servicio de Geriatría del Hospital Sociosanitari de L'Hospitalet SH/CSI. Nombre de la institución y dirección: Consorci Sanitari Integral (CSI). Av. Josep Molins, 29-41. 08906L'Hospitalet de Llobregat (Barcelona). Email: elisenda.reig@sanitatintegral.org

${ }^{2}$ Arias, R. Psicóloga Clínica adjunta Servicio de Psiquiatría del Hospital Moises Broggi/CSI

3 Vacas, M. Psicóloga Clínica adjunta Servicio de Psiquiatría del Hospital Moises Broggi/CSI

${ }^{4}$ Canadell,X. Psicólogo General Sanitario Servicio de Psiquiatría del Hospital Sociosanitari de L'Hospitalet $\mathrm{SH} / \mathrm{CSI}$

5 Prat, M. Psiquiatra del Hospital Moises Broggi/CSI
} 


\section{Introducción}

Durante la pandemia por Covid-19, diversos grupos de población (pacientes ingresados, familiares, y personal sanitario y/o no sanitario) presentaron sentimientos cuyo control fue complicado. El desconocimiento del virus, de cómo tratarlo, ha significado miles de muertos. En la primera ola, la capacidad sanitaria ha sido muy precaria y se ha visto desbordada, ya que el personal sanitario ha estado al borde del colapso, atendiendo a enfermos sin los medios ni los espacios ( $\sin$ camas de $\mathrm{UCI}$ ) suficientes. Paralelamente y desde fuera de los hospitales, las familias han estado sin la posibilidad de ver a sus familiares, sin tener noticias, y en algunos casos la única noticia que recibían era para anunciarles su muerte. Ya en la fase post-covid, las familias en pleno proceso traumático han tenido que enterrar a los suyos sin poder hacer los ritos funerarios indispensables para el duelo (ver al difunto ya muerto, rodearse de los familiares y amigos, hacer la ceremonia de despedida, etc..). Ante esta situación tan catastrófica, se hizo patente la necesidad de dar respuesta rápida a las familias de los pacientes ingresados que sufrían el desconsuelo de no poder estar con los suyos, a la vez que los profesionales sentían no sólo el sufrimiento de aquellos pacientes aislados en sus habitáculos, sino también el propio sentimiento de impotencia ante la falta de medios y de conocimiento respecto a cómo afrontar la cura de la enfermedad. La presencia de este estrés, vivido de manera sostenida, provocó reacciones severas de ansiedad.

La gran demanda de personas con sintomatología psiquiátrica nos hizo pensar en la posibilidad de realizar una atención grupal para los distintos sectores más afectados (personal trabajador de los Hospitales y centros del Consorcio Sanitario Integral (CSI), Médicos Internos Residentes (MIR), y Familias (en proceso de duelo).

¿Por qué una intervención grupal? Según Vinogradov y Yalom, la Terapia de Grupo es a día de hoy una terapia que tiene una ventajosa característica que es la disponibilidad. Se puede tratar a un elevado número de pacientes utilizando eficientemente el tiempo, el espacio, el personal y otros recursos. El grupo es más que un espacio de desarrollo y entrenamiento de la persona en acción, es un espació que le permite tener relación y reacción con los demás miembros del grupo. (J.M. Sunyer, 2008). Al mismo tiempo la cooperación consciente entre los miembros del grupo favorece su crecimiento conjunto (su realización). (J.Guimón, 2001).

Yalom (2000), describe once factores terapéuticos que operan en la terapia de grupo. El tener presente dichos factores fue uno de los motivos que nos llevó a la elección de este tipo de abordaje. Todos estos factores son elementos que suceden en todos los grupos y facilitan la mejora emocional y personal de los participantes 
Del total de los factores, en nuestras intervenciones resultaron especialmente relevantes:

Infundir esperanza: Observar la mejoría de otros compañeros y que los demás puedan observar la mejoría propia, infunde esperanza en la eficacia de la intervención. Se alcanza a través del testimonio de otros compañeros que están en otro momento más avanzado del proceso. (G. Duelo)

Universalidad: Muchas personas se sienten solas en sus desdichas, convencidas de que sólo ellos tienen ciertos problemas o sentimientos. En el grupo de terapia las personas experimentan un gran alivio cuando descubren que no están solos, que sus problemas son universales y los comparten otros miembros del grupo. (G. Duelo y G. Personal sanitario)

Altruismo: Para personas que se sienten desmoralizadas y creen no poder ofrecer nada de valor a nadie, la experiencia de poder ser útil a otros miembros del grupo puede resultar sorprendentemente gratificante, y aumentar además la autoestima. (G. Duelo)

Aprendizaje vicario: Durante la terapia los miembros se benefician de la observación de la terapia de otros integrantes que tienen problemas similares. (G. Duelo y $G$ Personal sanitario)

Intercambio de información es otra de las claves de esta terapia. Se trata de un intercambio muy enriquecedor, ya que a los participantes les resulta muy útil aprender más sobre otros usuarios.

Catarsis: En el grupo podemos experimentar en la propia piel que podemos expresar cualquier emoción y ser aceptado por los demás. Descubrimos que compartir nos hace ser aceptados por el grupo. Ser capaz de expresar emociones fuertes y profundas, y aun así ser aceptado por los demás, es algo que pone en entredicho que seamos inaceptables o que nadie pueda sentirse capaz de querernos. (G. Duelo y $G$ Personal sanitario).

Factores existenciales: Si bien el grupo ofrece la posibilidad de romper el aislamiento característico de la condición humana al mismo tiempo da pie al convencimiento de que el acompañamiento tiene un límite y guía el camino hacia la autorresponsabilidad y de la propia $\operatorname{soledad~(G.~Duelo)~}$

Cohesión: Es otra de las ventajas de este tipo de intervención. Los humanos por naturaleza, necesitamos formar parte de un grupo, tener un sentido de pertenencia y aceptación. Los miembros de un grupo cohesivo se aceptan y se apoyan mutuamente y tienden a formar relaciones significativas en el seno del grupo. (G. Duelo y $G$ Personal sanitario).

Cuando las personas están en crisis o en situación traumática, el grupo le permite compartir, aunque no lo verbalice, sentimientos, miedos vividos, a través de los otros. La cooperación, la matriz que establece el grupo, hace que se obtenga el beneficio de manera más rápida por el 
efecto espejo, observo, veo, como otro resuelve su problema y eso me facilita a mí, probar, cambiar, modificar aspectos personales y las relaciones que tengo con los demás y conmigo mismo.

Llevamos a cabo Terapia grupal con el objetivo de dar soporte psicológico a las familias de los pacientes ingresados, así como a las familias en proceso de duelo y a los profesionales (médicos, enfermeras, auxiliares de enfermería, administrativos, y otros servicios) se realizaron intervenciones en los diferentes centros asistenciales del Consorci Sanitari Integral (CSI). Éstas fueron llevadas a cabo por un grupo de profesionales de Salud Mental liderados desde la Unidad de Psiquiatría, en colaboración con el equipo de Recursos Humanos, el equipo de Trabajo Social y con el soporte de la Dirección del CSI y la participación voluntaria de 13 psicólogos más de la institución. Para ellos se creó un protocolo con título 'Suport psicosocial a professionals, pacients y familiars durant la crisi de la COVID-19' ("Soporte psicosocial a pofesionales, pacientes y familiares durante la crisid de la COVID-19").

Se llevó a cabo un protocolo donde se crearon dos equipos de intervención:

(1) Equipo de atención psicológica y psiquiátrica de urgencia, para realizar asistencia telefónica inmediata a los familiares de los pacientes ingresados y su posterior seguimiento, y también a los profesionales; en este segundo caso se creó el programa denominado "Ara et toca a tu" ("Ahora te toca a ti"), con el objetivo de llegar al máximo de personas atendidas. Aquellos profesionales que se sentían desbordados llamaban al número proporcionado por el programa. El equipo respondía mediante llamadas telefónicas, y, si se le detectaba sintomatología psiquiátrica, se le derivaba a atención individualizada, también telefónica, por parte de los psiquiatras del equipo. A los profesionales que no eran derivados al psiquiatra se les sugería la asistencia a la atención grupal. La detección de sintomatología psiquiátrica se realizaba mediante entrevista no estructura.

(2) Equipo de seguimiento de duelo, que realizaba asistencia telefónica a las $72 \mathrm{~h}$ de haber fallecido el familiar en cualquier centro del Consorci Sanitari Integral (CSI), para ayudar a crear un espacio de contención para los familiares que se sentían confusos, ansiosos, con sentimientos de culpa y de soledad, así como con impotencia, rabia, ira y mucho dolor.

En la fase más aguda de la pandemia, desde esta atención telefónica se evidenció una mayor necesidad de abordar las consecuencias psicológicas de la enfermedad, tanto en los profesionales como en los familiares de fallecidos por COVID. Los profesionales sanitarios y/o no sanitarios habían estado sometidos a un intenso estrés derivado de elevadas cargas de trabajo, falta de recursos, dudas éticas, miedo al contagio y aislamiento de la familia en algunos casos. Asimismo, en las familias atendidas por procesos de duelo por la muerte por 
COVID de algún familiar en alguno de nuestros centros, se detectaron casos en que el riesgo de duelo patológico era elevado.

Se planteó la posibilidad de organizar Grupos de soporte para dar mayor cabida a personas (profesionales/familiares) en situación de riesgo para su salud mental/emocional. Así pues, se diseñó un segundo proyecto de intervención 'Abordaje y afrontamiento del estrés en el post-COVID: Equipos de Protección Emocional (EPE)' por considerarse crucial facilitar espacios que ayudasen a gestionar el estrés y el malestar emocional vivido. Se prioriza el abordaje grupal para las diferentes intervenciones, al considerarlo más abarcativo y terapéutico.

El objetivo del estudio es explicar el proyecto de intervención Abordaje y afrontamiento del estrés en el post-COVID: Equipos de Protección Emocional (EPE) que el equipo de Psicología realizó en tres sectores poblacionales:

1. Programa Asistencial EPE para Profesionales del CSI dirigido a Personal Sanitario y no sanitario del Hospital Moisés Broggi (HMB), Hospital General de L'Hospitalet $(\mathrm{HGH})$, Centros Residenciales (Residencia Collblanc/ Residencia Francisco Padilla), Hospital Sociosanitario de L'Hospitalet (HSSH).

2. Programa Asistencial EPE para Médicos Residentes del CSI (R1, R2, R3, R4).

3. Programa Asistencial para familiares en Duelo

Fig 1. Programa de intervención Equipo de Protección Emocional (EPE)

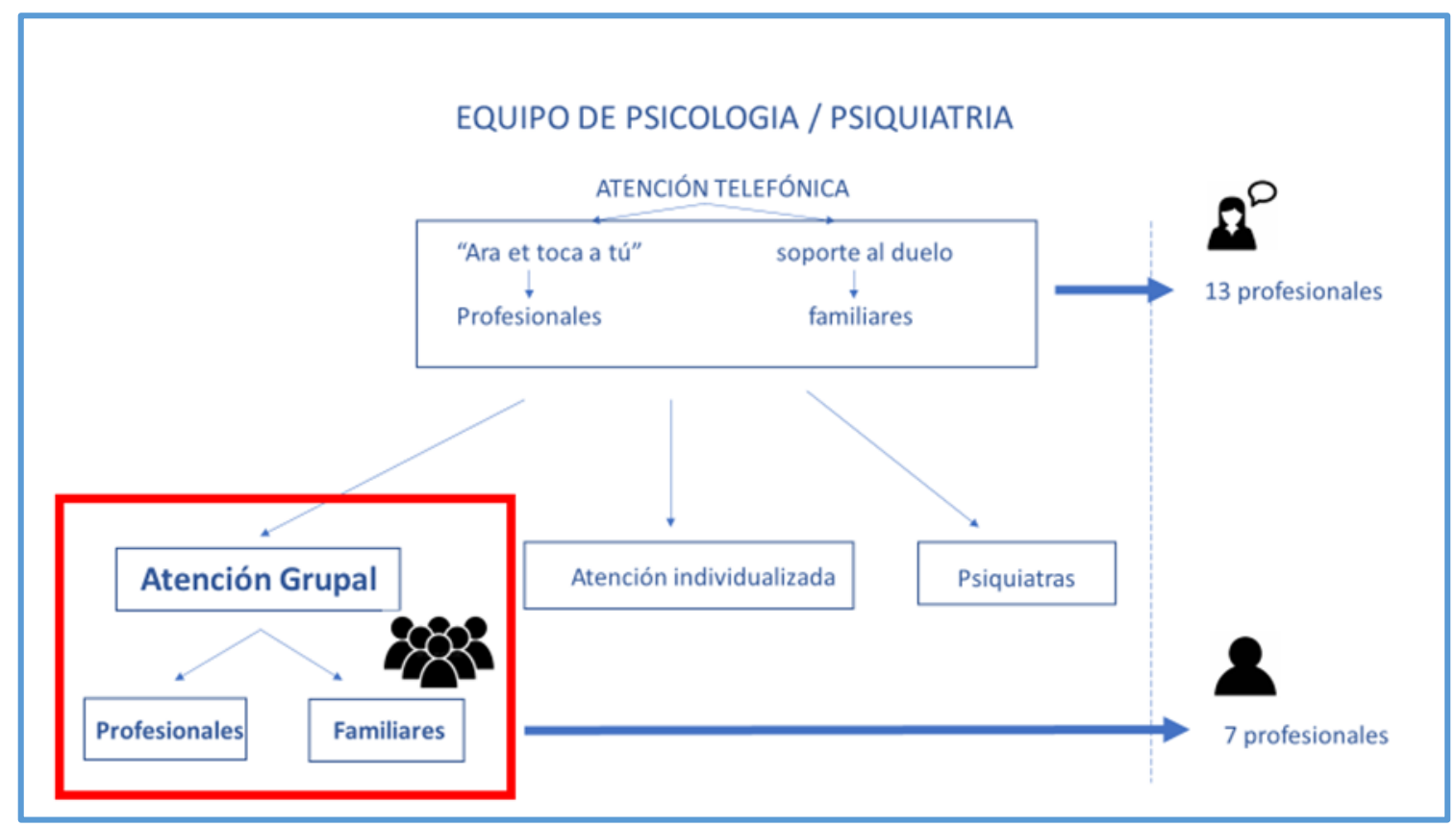


En la Figura 1, podemos ver la intervención global del equipo de psicología /psiquiatría. En este artículo nos centraremos en la intervención grupal que se llevó a cabo tanto a los profesionales de los centros asistenciales, a los grupos de Médicos internos residentes y a las familias que habían perdido a su familiar en alguno de nuestros centros (Hospitales, Residencias).

El objetivo del programa era implementar una intervención, que permitiese atender a un elevado número de personas, rápida, tanto en su aplicación como en la obtención de resultados, y efectiva para la contención y elaboración de los síntomas.

Para conseguir mayor efectividad en la intervención se consideró fundamental cumplir dos criterios:

1. Máxima rapidez en la aplicación del programa: está demostrado que la inmediatez en la aplicación del abordaje grupal aumenta su efectividad, ya que se consigue contener la sintomatología, se favorece el retorno a la normalidad y se hace prevención de la afectación traumática.

2. Máxima difusión del programa: los profesionales expuestos a un elevado estrés tienen una mayor dificultad para pedir soporte que la población general (Fridner, 2012) (Andrew, Louise; Brenner, B, 2015), actualmente se incrementa por la percepción de no haber llegado al final de la crisis, además de tener dificultades para identificar las señales de alarma. Por ello, se requirió el soporte de la institución que debía facilitar y fomentar la asistencia por parte del personal.

Así pues, además de intervenir en la gestión del estrés, se pretendió hacer una valoración de las posibles necesidades individuales, para poder redirigir aquellos profesionales o familiares a los recursos sanitarios existentes en caso de considerarse necesario. En el colectivo de los profesionales al PAIMM (programa atención integral al Médico enfermo de la Fundación Galatea), COPC (Colegio Oficial de Psicólogos de Cataluña). Y en el colectivo de los familiares a sus CAPs (Centros de Atención Primaria), CSMA (Centro de Salud Mental de Adultos), entre otros.

\section{Metodología}

En todos los grupos presenciales se mantuvieron las normas de seguridad preceptivas (uso de mascarilla, gel hidroalcohólico, distancia de 1,5-2m).

A continuación, iremos desarrollando la metodología de las tres intervenciones grupales a los distintos equipos/personas donde se llevó a cabo la intervención. 


\section{1.-Programa asistencial "Equipos de protección emocional" (EPE) para Profesionales del CSI}

Se contempló la intervención en los equipos de primera línea de los centros $\mathrm{HMB}, \mathrm{HGH}, \mathrm{HSSH}$, y de la unidad de Treball Social (TS), así como una intervención específica en las Residencias Francisco Padilla y Collblanc.

A partir del programa "Ara et toca a tu", se detectaron aquellas personas que requerían un soporte psicológico más exhaustivo porque habían sufrido una mayor sobrecarga asistencial durante la pandemia de la COVID-19. Se les informó/invitó a la participación en las sesiones de terapia grupal, cuya asistencia fue voluntaria.

\subsection{Objetivos del programa asistencial (EPE) para profesionales:}

- Identificar la sintomatología propia del estrés

- Estabilizar sintomatología emocional descompensada. Ventilación emocional, informar y normalizar las reacciones de estrés agudo, proporcionar recursos de autoprotección emocional, validar y fomentar sus estrategias de afrontamiento y resiliencia.

- Elaborar los incidentes críticos vividos (elevada mortalidad, elevada exposición al sufrimiento humano, acompañamiento en las fases finales, triaje de pacientes, responsabilidad asumida por profesionales de otras especialidades...)

- Prevenir la agudización de la sintomatología

\subsection{Metodología:}

- Intervención: formato grupal

- Duración: 90 minutos por sesión. Las sesiones se repitieron entre lunes y jueves desde las 9 de la mañana hasta las 18/20.30h, para facilitar la máxima asistencia.

- Número de participantes/conductores: 10-12 personas por sesión, para dar viabilidad al trabajo grupal y para poder adaptar los espacios manteniendo la distancia de seguridad recomendada. Un conductor.

- Período: 1 de junio al 3 de julio (5 semanas de duración).

\subsection{Recursos:}

- Profesionales: 4 Psicólogos. Dos de la propia empresa y dos psicólogos externos especialistas en Técnicas de emergencias. 
- Espacios: Se dispuso de espacios en los diferentes centros donde se garantizaba la intimidad para así facilitar que los profesionales pudieran encontrarse a gusto.

\section{2.- Programa Asistencial (EPE) para Médicos Residentes del CSI}

En el grupo de Médicos Internos Residentes (MIR), al estrés habitual que comporta la propia residencia, se añadió el de la actual pandemia, que como se ha mencionado, sometió a todo el sistema sanitario a un elevado estrés. El programa se orientó a dar soporte y cuidar de la salud mental de este colectivo que se vio expuesto a una gran tensión emocional y a unas exigencias excepcionales que sobrepasaban los recursos disponibles.

\subsection{Objetivos (los mismos que los objetivos de los profesionales):}

- Tomar conciencia de los síntomas propios del estrés

- Ventilación y estabilización emocional

- Dotar de estrategias de afrontamiento

- Detectar casos que requieran atención individualizada o derivación (PAIMM)

\subsection{Metodología:}

- Intervención: formato grupal, cada persona asistía a cuatro sesiones, una por semana.

- Duración: Cada sesión constaba de dos partes de 60 min (debriefing + mindfulness) cada una con un intervalo de unos 30 min entre ellas.

- Número de participantes/conductores: 10 personas por sesión, más dos conductores por sesión.

- Período: 1 de junio al 3 de julio (5 semanas de duración).

\subsection{Recursos humanos y espacios:}

- Profesionales: 3. Un médico Internista / Una psicóloga y psiquiatra6

- Espacios: Se dispuso de espacios en los diferentes centros donde se garantizaba la intimidad para así facilitar que los profesionales pudieran encontrarse a gusto.

\footnotetext{
${ }^{6}$ En formación en Terapia Analítica Grupal que participó de forma voluntaria 


\section{3.- Programa asistencial EPE para familias en Duelo.}

En la atención telefónica a aquellos familiares que habían sufrido la pérdida de un ser querido, se llevó a cabo una selección por cribaje (entrevista no estructurada) de aquellas personas que necesitaban un soporte psicológico más exhaustivo. Se planteó una intervención breve, dada la disponibilidad de tiempo y recursos.

\subsection{Objetivos:}

- Mitigar la sensación de aislamiento y soledad.

- Favorecer la expresión de las emociones y normalizarlas

- Detectar nivel de riesgo y síntomas predictores de complicaciones de duelo.

- Facilitar derivación a recurso específico en caso de que fuera necesario (CSMA, CAP, Profesionales privados)

\subsection{Metodología:}

- Intervención: formato grupal, cada persona asistía a cuatro sesiones, una por semana.

- Técnica: Sesiones semiestructuradas de Terapia Grupal (TG) Grupoanalítica.

- Duración: Cada sesión constaba de 60 min

- Número de participantes/conductores: 10 personas por sesión, más dos conductores por sesión. Se realizó un total de 4 grupos, tres de los cuales fueron presenciales y un grupo online. Se distribuyeron dependiendo del vínculo con el familiar fallecido y las posibilidades de manejo de la plataforma virtual

Grupos Presenciales:

Orfandad (grupo A)

Viudedad (grupo C)

Orfandad (grupo D)

Grupos Online: Jóvenes en duelo (grupo B)

Para conducir el grupo online con la máxima confidencialidad y con las normas de grupo, tan importantes para garantizar el buen funcionamiento del mismo, se les envió un documento donde los participantes se comprometían a la confidencialidad, la confianza y la discreción de las sesiones.

- Período: 1 de junio al 3 de julio (5 semanas de duración).

\subsection{Recursos y espacios}

- Profesionales: 2 Psicólogos. 
- Espacios: Se dispuso de espacios en los diferentes centros donde se garantizaba la intimidad para así facilitar que los profesionales pudieran encontrarse a gusto.

\section{$\underline{\text { Resultados }}$}

Nos parece interesante separar los resultados según el sector poblacional en el que se ha intervenido.

\section{1.- Programa Asistencial EPE para Profesionales}

A pesar de que el programa contemplaba la realización de sesiones para aquellos servicios que primero mostraron altos niveles de estrés: UCl, urgencias, Unidades de Hospitalización, Trabajo Social y la dos Residencias del CSI, una vez iniciado el programa se recibió la petición y la demanda de otros servicios (Rehabilitación, HADO (Hospitalización a domicilio), diagnóstico por la imagen, y camilleros. A todos ellos se les atendió con el mismo programa de cinco semanas dado que empezaban el período vacacional.

El programa EPE de soporte grupal atendió a un total de $\mathrm{N}=392$ profesionales. Posteriormente, se realizaron 44 intervenciones individuales, de las cuales 13 habían asistido a la sesión grupal, evidenciando la necesidad de una mayor atención psicológica y emocional individualizada. De igual modo, algunos profesionales lo solicitaron al final del grupo.

En las gráficas podemos observar la participación de profesionales (Fig. 2): enfermería (DUI) un 44\%; Técnicos en Cuidados auxiliares de enfermería (TCAI) un 25,5\%; Facultativos 15,5\%; Trabajo Social 6,6\%; otros (administrativos/camilleros) 8\%

Y por centros del CSI (Fig. 3): Hospital Moisés Broggi 36\%; Hospital General de L'Hospitalet 7\%; Hospital Sociosanitario 1\%; Residencias 4\%; Trabajo Social 4\%.

Fig. 2: Participación de Profesionales

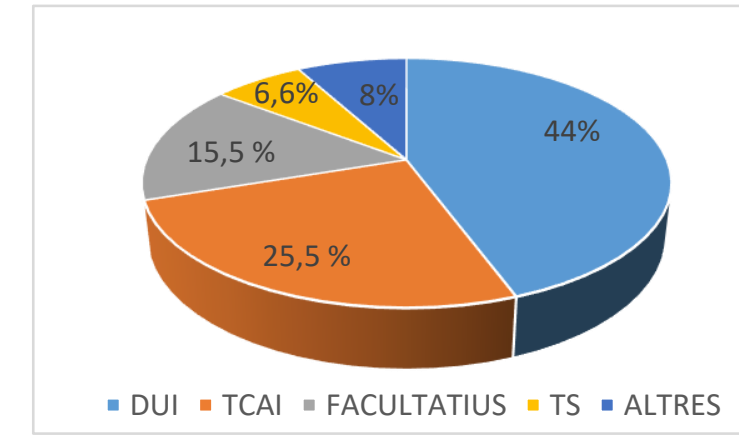

Fig. 3: Distribución por Centros del CSI

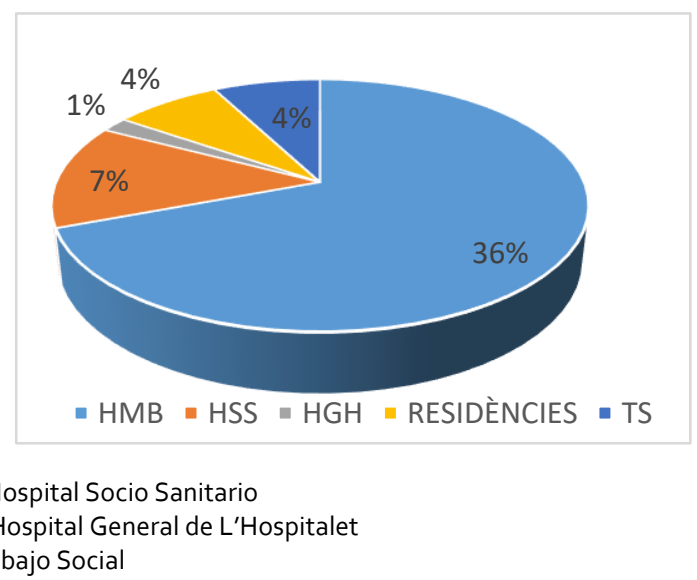

DUI.= Unidad de Enfermeria $\quad \mathrm{HMB}=$ Hospital Moises Brogg

TCAI. = Técnicos en Cuidados auxiliares de enfermería HSS= Hospital Socio Sanitario

Facultativos (Médicos de diversas especialidades) $\quad$ HGH= Hospital General de L'Hospitalet

TS= Trabajo Social

TS= Trabajo Social 
Hemos de destacar que el Hospital Moises Broggi (HMB), es el Hospital más grande del CSI y por tanto estos factores eran más evidentes que en los otros hospitales, ya que es donde llegaban la mayoría de los enfermos y donde no se daba cabida a toda la población infectada. Además, el número de profesionales del HMB es muy superior a los otros centros, con lo que la demanda de soporte psicológico siempre fue mayor. Como apunte diremos que en el mismo momento de la crisis del COVID, la gravedad del personal asistencial era tan alta, que se llevaron a cabo algunas sesiones grupales en la misma sala de urgencias del HMB, para poder contener, aunque fuera levemente, el estrés que vivían los profesionales.

Desde la psicología de emergencias se ha fundamentado experimentalmente la afectación del estrés a causa de situaciones de crisis. La situación actual, con muchos factores estresantes que confluyen en un mismo momento, tiene un factor que la hace potencialmente de riesgo, y es el estrés sostenido durante un largo periodo de tiempo. Si inicialmente ese estrés actuó como un potenciador, podríamos decir que a la larga ha generado un elevado agotamiento en el personal.

Un suceso se vuelve más estresante cuanto más cuesta desconectar de este suceso, en este caso, la falta de conocimiento respecto al virus y la sobreinformación y infotoxicidad a la que han estado sometidos los profesionales, ha hecho muy complicado desconectar durante las primeras semanas.

Aunque los profesionales sanitarios son personas que trabajan bien bajo presión, la situación de desconocimiento de este virus ha modificado el sentido de control con el que se trabaja habitualmente, generando un sentimiento de amenaza y constante estado de alerta.

\section{2.- Programa Asistencial EPE para Médicos Residentes del CSI}

El programa se ofertó a los 60 residentes del Consorcio Sanitario Integral, siendo la participación voluntaria, lo que representó un $47 \%$ del total de residentes.

Se realizaron tres grupos, en los que participaron un total de 28 (Médicos Internos Residentes de las especialidades Medicina Interna (MIR), Cirugía ortopédica y traumatología (COT), Cirugía, Anestesia, Geriatría y Medicina Familiar y Comunitaria), de los cuales fueron 23 mujeres ( $82 \%)$ y 5 hombres (18\%).

En el Trabajo grupal compartieron sus emociones, se reconocieron como humanos, con miedo, cansancio, frustración, pero también con muchas herramientas para superar la crisis sanitaria. Expresaron como sentían haber madurado personal y profesionalmente. Valoraron 
como aprendizajes derivados de la pandemia: el mejor manejo de la incertidumbre y la capacidad de trabajar en equipo.

Se realizaron encuestas de valoración, en las que puntuaron la utilidad del taller con un 8,85 sobre 10 , el $90 \%$ haría una segunda sesión, el $86 \%$ lo implantaría de forma fija y el $100 \%$ lo recomendaría a otros compañeros. Algunas observaciones cualitativas permiten comprender la necesidad que existía de este programa como herramienta psicológica para el personal sanitario más joven y en formación: "Una sesión cómo ésta al mes para todo el personal sanitario, debería ser obligatoria para trabajar más y más felices"

"Estos talleres han sido una tabla de salvamento emocional en medio de la tempestad COVID. Ojalá contáramos con más de ellas para otras tormentas de la residencia"

\section{3.- Programa Asistencial para familiares en Duelo}

En la primera etapa de la pandemia, el equipo del soporte al duelo llevó a cabo 335 llamadas telefónicas en el periodo comprendido entre el 23 de marzo 2020 hasta el 15 de mayo 2020. Del total de llamadas, 105 fueron llamadas de seguimiento a aquellos familiares en que se detectaron síntomas de ansiedad, rabia, ira, sentimientos que enmascaran una profunda tristeza y/o depresión y aquellas personas donde se podía prever la aparición de cuadros psiquiátricos de difícil resolución. La mayoría de los casos presentaban sintomatología ansiosa, ansiedad anticipatoria, insomnio, ansiedad ante la muerte, soledad en la toma de decisiones, ideas catastróficas, culpa del contagio familiar, agotamiento.

Entre los diversos grupos se atendió un total de $\mathrm{N}=29 ; 20$ personas en las sesiones presenciales y 9 en el formato on-line. Se llevó a cabo una selección exhaustiva de los participantes. Del total el $80 \%$ eran mujeres y el $20 \%$ hombres. Se pudieron configurar grupos muy homogéneos, los grupos A y D, estaban formados exclusivamente por hijas/os, el grupo $B$ por hijos jóvenes (que fue el grupo on-line) y el grupo $C$ exclusivamente por viudas. El $73 \%$ del total de participantes eran hijos/as que habían perdido a uno o a los dos padres, es decir habían quedado huérfanos, mientras que el $27 \%$ fueron viudas. En los grupos $A$ y $C$, hubo personas que fueron las que contagiaron a sus familiares, lo que generó grandes sentimientos de culpa.

En los grupos de duelo, observamos que los familiares se mostraron muy receptivos a recibir ayuda cuando se les propuso la participación en los grupos. En las primeras sesiones los sentimientos más frecuentes fueron la impotencia y la rabia ante la falta de información y el descontrol vivido en los hospitales (haciendo referencia a la pérdida de objetos personales, y para ellos valiosos), así como la desinformación respecto a la evolución y pronóstico de su 
familiar. El desconocimiento de la enfermedad provocó situaciones sorprendentes para los propios médicos, con casos de pacientes que evolucionaron favorablemente y en cuestión de horas, después de un distrés global se producía el fallo respiratorio y muerte. Situaciones de este tipo han sorprendido a profesionales y a los familiares les ha dejado con un sentimiento de haberse sentido engañados. Otros familiares relataban muchos sentimientos de tristeza y dolor, ante la sorpresa de la muerte, la imposibilidad de despedirse, así como la soledad después del fallecimiento.

Una queja que apareció frecuentemente fue en relación a la dificultad de realizar los rituales tan necesarios para poder despedir a los familiares, con lo que el sentimiento de estar viviendo algo irreal todavía fue más evidente. A medida que se avanzaba en las sesiones grupales, los pacientes iban cambiando sus sentimientos de rabia/ira, hacia la tristeza, permitiéndose conectar más con el vacío y la pérdida.

El denominador común de casi todos los participantes fue la falta de ritos de despedida, de ver a su ser querido, del acompañamiento de los familiares, algunos de los cuales no habían podido recoger las cenizas y se encontraban como en una situación de desconcierto y de irrealidad, en estado de choque. Según Payàs (2016), "el estado de choque tiene una función anestésica, que nos da tiempo y una cierta serenidad que nos permite hacer frente a lo que vendrá después del entierro". Para los participantes en este grupo de duelo para familiares, este tiempo de crisis covid-19 fue una pesadilla, porque no sabían ni donde estaba su familiar, ni cuándo podrían recogerlo y proceder al entierro pertinente. Cuando se producía el entierro, éste tenía además la característica de ser rápido y con solo 3 personas, con lo que el sentimiento de soledad y desasosiego todavía fue mayor. Se consideró importante hacer hincapié en este hecho, el no haber podido hacer ningún rito de despedida. Por ello se diseñó una última sesión en que se les pedía, que trajeran una carta de despedida y se proponía leerla y compartirla con el grupo a modo de "ritual". La mayoría redactaron la carta y posteriormente la leyeron a sus compañeros. Fueron momentos cargados de una gran intimidad, donde cada hija/o y/o viuda, pudo compartir con el resto, recuerdos, emociones, sentimientos, que de alguna manera u otra todos tenían, y pudieron llorar, acompañándose en ese momento tan suyo. Fueron testimonios muy sinceros y auténticos, lo que permitió a aquellos que no habían escrito la carta, llorar también por los suyos.

Expresaron sentimientos de gratitud y se dio una rápida cohesión entre ellos, al sentirse comprendidos e identificados con el resto, por haber vivido situaciones muy parecidas a las suyas. Ello minimizó los sentimientos de soledad en los que se veían sumergidos durante toda la pandemia. 
La valoración de los psicólogos conductores implicados en estas 4 sesiones fue muy positiva, considerándolas muy provechosas, aun siendo conscientes de la brevedad de la intervención por lo expuesto anteriormente.

La necesidad de estos grupos de duelo, así como la demanda de continuar con ellos, fue prácticamente unánime y no se descarta retomarlos en septiembre si la situación sanitaria lo permite, teniendo en cuenta que se debería dar una mayor duración para poder también dar cabida a todas las personas que han solicitado ayuda psicológica. (Tabla 1)

Tabla 1.- Cuadro de las intervenciones Grupales en los 3 colectivos

\begin{tabular}{|l|l|l|l|}
\hline Selección Previa & Personal sanitario & MIR & Familiares \\
\hline Heterogeneidad & Muy elevada +++ & Baja + & $\begin{array}{l}\text { Fase de screening } \\
\text { telefónico }\end{array}$ \\
\hline $\begin{array}{l}\text { Vulnerabilidad } \\
\text { Número } \\
\text { profesionales de }\end{array}$ & $\begin{array}{l}\text { Baja }+ \text { por perfil } \\
\text { formación }\end{array}$ & $\begin{array}{l}\text { Alta }+++ \\
\text { Por perfil personal } \\
\text { e inexperiencia }\end{array}$ & $\begin{array}{l}\text { Alta }+++ \\
\text { por falta de decursos } \\
\text { emocionales }\end{array}$ \\
\hline $\mathbf{N}$ & 392 profesionales & 28 MIRs & 2 psicólogos \\
\hline
\end{tabular}

\section{Discusión}

El desbordamiento asistencial en el que se ha encontrado el sistema sanitario en esta pandemia ha provocado un alto nivel de estrés para todos aquellos que han estado al frente de la situación. Factores como la gravedad de los casos, la exposición, la sobrecarga, la falta de recursos, el miedo al contagio, la elevada mortalidad, la complejidad y la incertidumbre, son algunos de los factores de estrés identificados. El nivel de estrés y su afectación está en consonancia con la responsabilidad de cada profesional, la sobrecarga laboral y factores de personalidad.

Se pueden concluir algunos aspectos comunes en todos los grupos llevados a cabo, independientemente fueran profesionales o familias.

Los aspectos comunes de las tres intervenciones, a profesionales, a Médicos internos residentes y a los familiares de pacientes fallecidos, serían: 
A.- Presencia de sintomatología de estrés agudo. Siendo el más frecuente: la fatiga por compasión, los trastornos del sueño, ansiedad, afectación anímica, dificultades en los niveles de atención y concentración, sintomatología neurovegetativa, irritabilidad, imágenes intrusivas de los sucesos traumáticos.

B.- Aumento en la cohesión de los participantes del grupo (fuera cual fuera) después de compartir experiencias, sentimientos, emociones. Se debe poner especial relevancia en la observación de estrategias muy adaptativas, así como afrontamientos muy efectivos, especialmente en los grupos de profesionales donde ya previamente había mucha cohesión. La cohesión actúa como factor protector y de contención, siendo menos necesaria la intervención psicológica individualizada. Es destacable que este mismo hecho se identificó en los grupos de familiares, que siendo personas desconocidas llegaron a cohesionarse en tan solo 4 sesiones y consiguieron sentirse acompañados por personas que habían vivido lo mismo, o situaciones similares.

C.- aumento de la empatía entre los participantes, por el mismo motivo que la cohesión, el compartir situaciones parecidas, hace que las personas, entendamos más a los otros, porque han vivido algo parecido.

D.- Aumento de Tolerancia a la frustración. Cuando vemos las dificultades de nuestros coetáneos /compañeros, y vemos otras maneras de afrontar o no los problemas, somos capaces de exigirnos menos, de juzgarnos menos y de poder afrontar el estrés de una manera más saludable. (Martin Ausero, 2011).

La valoración del programa es satisfactoria. Aun sabiendo que estas sesiones no son suficientes para evitar que los participantes (personal sanitario y/o no sanitario y familias) dejen de experimentar estrés, se puede ayudar a reducirlo, contenerlo y limitar sus efectos devastadores.

La situación a la que se ha enfrentado el sistema sanitario genera muchas preguntas y dudas a nivel médico, y desde la psicología, se dispone de estudios que dan respuestas a cómo afecta el estrés en situaciones de crisis, también se plantean nuevas preguntas dada la excepcionalidad de lo que estamos viviendo. Existe mucha incertidumbre sobre cómo acabará afectando el estrés sostenido en los profesionales, el confinamiento en los ciudadanos, las pérdidas de familiares sin poder hacer los ritos de despedida y duelo, la situación económica que vendrá, y muchas otras cuestiones que siguen pendientes. Es por esto por lo que basándonos en el conocimiento existente y dada la incertidumbre actual, se valora que la Terapia Grupal es la mejor opción en este momento, en relación a la salud mental, ya que ayuda a gestionar e integrar lo vivido, esperando que actúe como preventivo 
al dotar a los profesionales de estrategias de gestión que permitan afrontar la incertidumbre en la que seguimos inmersos como sociedad.

Concluimos que:

- El abordaje grupal es una alternativa terapéutica válida y especialmente útil en momentos de pandemia como el actual.

- La terapia grupal abarca más dimensiones y posee un potencial terapéutico enorme: Facilita la identificación con "iguales" mediante la resonancia, posibilita el consejo pedagógico de otros compañeros y observar diferentes estrategias de afrontamiento y rescata del aislamiento (Foulkes, 2015).

Desde el punto de vista de la gestión de los centros sanitarios asistidos, es interesante tener en cuenta el gran número de personas asistidas ( $N=449$ personas) que se han podido beneficiar del programa con una intensidad de recursos profesionales relativamente baja (7 psicólogos y un psiquiatra).

Se puede considerar que la terapia grupal es una gran alternativa, tanto por sus beneficios individuales para cada uno de los participantes, garantizando cambios personales, a la vez que permite la adquisición de técnicas de afrontamiento, como para las instituciones hospitalarias, a las cuales permite dar ayuda a un gran número de personas, con un excelente coste-beneficio, en una situación de emergencia como la vivida en la crisis del COVID-19.

\section{REFERENCIAS}

Andrew, L.; Brenner, B. (2015). Psysician Suicide. Medscape. Vol 17.

Cano-Langreo M; Cicirello-Salas S; López-López A.et al. (2014). Marco actual del suicidio e ideas suicidas en personal sanitario. Medicina y Seguridad del Trabajo 6o(234) 198-218 DOI: $10.4321 / 50465-546 \times 2014000100015$

Foulkes, S.H; Anthony, E.J. (2006). Psicoterapia de Grupo. El enfoque Psicoanalítico. Gráficas Rey. S.L. Barcelona.

Foulkes, S.H. (2015). Psicoterapia Grupoanalítica. Método y principios. Ed. Lozano-Faisano. Barcelona.

Fridner,A. Belkic,K. Marini,M. et al. (2012). Why don't academic physicians seek needed professional help for psychological distress?. Swiss Medical Weekly 142 (JULY) DOI: $10.4414 / \mathrm{smw} .2012 .13626$

Guimón,J. (2001). Manual de Terapias de Grupo. Tipos, Modelos y Programas. Biblioteca Nueva. Madrid.ISBN:978-84-9742-113-3 
Maestre, J.M; Rudolph, J.W. (2015). Theories and Styles of Debriefing: the Good Judgment Method as a Tool for Formative Assessment in Healthcare. Rev. Española de Cardiología. Vol. 68. Núm. 4. páginas 282-285

Martín Asuero, A. (2016). Con rumbo propio. Disfruta de la vida sin estrés. Ed.Plataforma Editorial. Barcelona.

Payàs Puigarnau, A. (2016). El mensaje de las lágrimas. Una guía para superar la pérdida de un ser querido. Editorial Paidós Divulgación. Barcelona.

Rigg,I.; McColl,J. (2017). Mindfulness based living. An exploratory study with health care staff. Dialnet, ISBN 978-84-617-9388-4, págs. 153-173

Sunyer Martín, J.M. (2008). Psicoterapia de Grupo Grupoanalítica. La co-construcción de un conductor de grupos. Biblioteca Nueva. Madrid.ISBN: 978-84-9742-894-1

Vinogradov, S.; Yalom, I.D. (1996). Guía breve de psicoterapia de grupo. Paidós. Barcelona. ISBN: 978-84-493-0224-4

Yalom, Irvin D. (1984). Psicoterapia Existencial. Herder Barcelona.

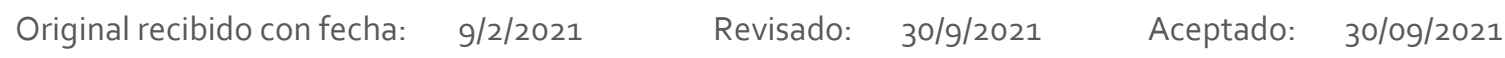

